\title{
Bäcklund Transformation and Quasi-Periodic Solutions for a Variable-Coefficient Integrable Equation
}

\author{
Wenjuan Rui and Yufeng Zhang \\ College of Science, China University of Mining and Technology, Xuzhou 221116, China \\ Correspondence should be addressed to Yufeng Zhang; zhangyfcumt@163.com
}

Received 12 March 2014; Accepted 27 April 2014; Published 18 May 2014

Academic Editor: Tiecheng Xia

Copyright (c) 2014 W. Rui and Y. Zhang. This is an open access article distributed under the Creative Commons Attribution License, which permits unrestricted use, distribution, and reproduction in any medium, provided the original work is properly cited.

Binary Bell polynomials are applied to construct bilinear formalism, bilinear Bäcklund transformation, Lax pair, and infinite conservation laws of the generalized variable-coefficient fifth-order Korteweg-de Vries equation. In the meantime, quasi-periodic wave solutions for the equation are obtained by using the Riemann theta function. The asymptotic properties of one-periodic wave solution and two-periodic wave solutions are also established, respectively.

\section{Introduction}

Nonlinear evolution equations (NLEEs) have attracted intensive attention in the past few decades, since they occur in a variety of physical applications. It is always important to search for explicit and exact solutions. Various kinds of exact solutions such as soliton, peakon, complexiton, rational, periodic, and quasi-periodic solutions have been presented for NLEEs. Successful methods include the inverse scattering method [1], the Darboux transformation [2-4] and the Bäcklund transformation $[5,6]$, the Hirota method $[7,8]$, and algebrogeometrical approach [9-11]. Among the abovementioned methods, the Hirota method is a powerful approach to construct exact solution of nonlinear equations. By applying the Hirota method, people obtained a series of multisoliton solutions and rational solutions of many nonlinear equations in a systematic way. Unfortunately, this method relies on particular skills, appropriate exchange formulas, and complex calculations. On the other hand, in recent years, Lambert, Gilson et al. proposed an alternative procedure based on the use of the Bell polynomials to obtain parameter families of bilinear Bäcklund transformation and Lax pairs for soliton equations in quick and short way [12-14]. Fan developed this method to find infinite conservation laws of soliton equations [15-17] and proposed the super Bell polynomials
$[18,19]$. Ma systematically analyzed the connection between Bell polynomials and new bilinear equations [20].

From bilinear forms, Nakamura proposed a convenient way to construct a kind of quasi-periodic solutions of nonlinear equation in his two serial papers [21,22], where the quasi-periodic wave solutions of the KdV equation and the Boussinesq equation were obtained by using the Riemann theta function. Recently, Hon et al. have extended this method to investigate the discrete Toda lattice [23], $(2+1)$ dimensional Bogoyavlenskiis breaking soliton equation [24], and the asymmetrical Nizhnik-Novikov-Veselov equation [25]. Ma et al. constructed one-periodic and two-periodic wave solutions to a class of $(2+1)$-dimensional Hirota bilinear equations [26]. Zhang et al. applied this method to get periodic wave solutions of the variable-coefficient $\mathrm{mKdV}$ equation [27].

Due to the inhomogeneities of media and nonuniformities of boundaries in various real physical situations, the variable-coefficient NLEEs are considered to be more realistic than constant-coefficient equations in describing a large variety of real phenomena; for example, many physical and mechanical situations are governed by variablecoefficient KdV equation, for example, the nonlinear excitations of a Bose gas of impenetrable bosons with longitudinal 
confinement, the nonlinear waves in types of rods [2830]. Obviously, equations with variable-coefficient are much more complicated than constant-coefficient forms, and much attention has been paid to this subject [31-35]. In this paper, we will focus our study on the generalized variable-coefficient fifth-order Korteweg-de Vries equation such as the one given below:

$$
\begin{gathered}
u_{\mathrm{t}}+a(t) u u_{x x x}+b(t) u_{x} u_{x x}+c(t) u^{2} u_{x}+d(t) u u_{x} \\
+e(t) u_{x x x}+l(t) u_{x x x x x}+m(t) u+n(t) u_{x}=0,
\end{gathered}
$$

where $u$ is a function of $x$ and $t$ and $a(t), b(t), c(t), d(t)$, $e(t), l(t), m(t)$, and $n(t)$ are analytic functions of $t$. Since there are choices for the parameters, the variable-coefficient NLEEs can be considered as generalizations of the constant coefficient ones. Under certain constraint conditions, the variable-coefficient models may be proved to be integrable and given explicit analytic solutions [36]. The corresponding constraint conditions on (1) in this paper, which are obtained by the Painlev analysis [37] and conditions from the variablecoefficient models mapped to the completely integrable constant-coefficient counterparts [38], will be

$$
\begin{gathered}
a(t)=b(t)=\frac{15 l(t)}{\rho} e^{\int m(t) d t}, \quad c(t)=\frac{45 l(t)}{\rho^{2}} e^{\int 2 m(t) d t}, \\
d(t)=e(t)=0,
\end{gathered}
$$

where $\rho \neq 0$ is an arbitrary constant. The main goal of this paper is twofold. First, we apply the binary Bell polynomials to construct bilinear formalism, bilinear Bäcklund transformation, Lax pairs, and infinite conservation laws of (1) under condition (2). Second, we obtain the periodic wave solutions of the equation by using the Riemann theta function and discussing their asymptotic properties.

The organization of this paper is as follows. In Section 2, we briefly present necessary notations on binary Bell polynomial that will be used in this paper. In Section 3, we get bilinear formalism, bilinear Bäcklund transformation, Lax pairs, and infinite conservation laws of the generalized variable-coefficient fifth-order Korteweg-de Vries equation by utilizing the binary Bell polynomials. In Section 4, we apply Hirota's bilinear method to construct one- and twoperiodic wave solutions (1), respectively. Further we use a limiting procedure to analyze asymptotic behavior of the periodic wave solutions in detail. Finally, some conclusions are given in Section 5.

\section{Binary Bell Polynomials}

To begin with, we will give some basic concepts and notations about the Bell polynomials. For details, please refer to [11-13].

Let $f=f\left(x_{1}, x_{2}, \ldots, x_{l}\right)$ be a $C^{\infty}$ function with multivariables; the following polynomials

$$
\begin{aligned}
Y_{n_{1} x_{1}, \ldots, n_{l} x_{l}}(f) & =Y_{n_{1}, \ldots, n_{l}}\left(f_{r_{1} x_{1}, \ldots, r_{l} x_{l}}\right) \\
& =\exp (-f) \partial_{x_{1}}^{n_{1}} \cdots \partial_{x_{l}}^{n_{l}} \exp (f)
\end{aligned}
$$

are called the multidimensional Bell polynomials, where

$$
f_{r_{1} x_{1}, \ldots, r_{l} x_{l}}=\partial_{x_{1}}^{r_{1}} \ldots \partial_{x_{l}}^{r_{l}} f, \quad r_{1}=0, \ldots, n_{1}, \quad r_{l}=0, \ldots, n_{l} .
$$

For convenience, we denote the multidimensional Bell polynomials by $Y$-polynomials.

For example, for the simplest case $f=f(x)$, the onedimensional Bell polynomials are

$$
\begin{aligned}
& Y_{1}=f_{x}, \quad Y_{2}=f_{2 x}+f_{x}^{2}, \\
& Y_{3}=f_{3 x}+3 f_{x} f_{3 x}+f_{x}^{3}, \ldots
\end{aligned}
$$

For $f=f(x, t)$, the two-dimensional Bell polynomials are

$$
\begin{aligned}
Y_{x}(f) & =f(x), \\
Y_{2 x}(f) & =f_{2 x}+f_{x}^{2}, \\
Y_{2 x}(f) & =f_{3 x}+3 f_{x} f_{2 x}+f_{x}^{3}, \\
Y_{x, t}(f) & =f_{x, t}+f_{x} f_{t}, \\
Y_{2 x, t}(f) & =f_{2 x, t}+f_{2 x} f_{t}+2 f_{x, t} f_{x}+f_{x}^{2} f_{t}, \ldots
\end{aligned}
$$

Based on the above Bell polynomials, the multidimensional binary Bell polynomials ( $\mathscr{Y}$-polynomials) can be defined as follows:

$$
\begin{aligned}
\mathscr{Y}_{n_{1} x_{1}, \ldots, n_{l} x_{l}}(v, w) & =\left.Y_{n_{1} x_{1}, \ldots, n_{l} x_{l}}(f)\right|_{f_{r_{1} x_{1}, \ldots, r_{l} x_{l}}} \\
& = \begin{cases}v_{r_{1} x_{1}, \ldots, r_{l} x_{l}}, & r_{1}+\cdots+r_{l} \text { is odd, } \\
w_{r_{1} x_{1}, \ldots, r_{l} x_{l}}, & r_{1}+\cdots+r_{l} \text { is even. }\end{cases}
\end{aligned}
$$

The $\mathscr{Y}$-polynomials inherit the easily recognizable partial structure of the Bell polynomials. The first few lowest order binary Bell polynomials are

$$
\begin{aligned}
y_{x}(v) & =v_{x}, \quad y_{2 x}(v, w)=w_{2 x}+v_{x}^{2}, \\
y_{x, t}(v, w) & =w_{x, t}+v_{x} v_{t}, \\
y_{3 x}(v, w) & =v_{3 x}+3 v_{x} w_{2 x}+v_{x}^{3}, \\
y_{4 x}(v, w) & =w_{4 x}+3 w_{2 x}^{2}+4 v_{x} v_{3 x}+6 v_{x}^{2} w_{2 x}+v_{x}^{4}, \\
y_{5 x}(v, w)= & v_{5 x}+5 v_{x} w_{4 x}+10 v_{3 x} w_{2 x}+10 v_{x}^{2} v_{3 x} \\
& +15 v_{x} w_{2 x}^{2}+10 v_{x}^{3} w_{2 x}+v_{x}^{5} .
\end{aligned}
$$

Theorem 1 (see [11]). The link between binary Bell polynomials $\mathcal{Y}_{n_{1} x_{1}, \ldots, n_{l} x_{l}}(v, w)$ and the standard Hirota bilinear equation $D_{x_{1}}^{n_{1}} \cdots D_{x_{l}}^{n_{l}} F \cdot G$ can be given by an identity

$$
\begin{gathered}
\mathscr{Y}_{n_{1} x_{1}, \ldots, n_{l} x_{l}}\left(v=\ln \frac{F}{G}, w=\ln F G\right) \\
=(F G)^{-1} D_{x_{1}}^{n_{1}} \cdots D_{x_{l}}^{n_{l}} F \cdot G
\end{gathered}
$$


in which $n_{1}+n_{2}+\cdots+n_{l} \geq 1$ and operators $D_{x_{1}} \cdots D_{x_{l}}$ are classical Hirotas bilinear operators defined by

$$
\begin{aligned}
D_{x_{1}}^{n_{1}} \cdots & D_{x_{l}}^{n_{l}} F \cdot G \\
= & \left(\partial_{x_{1}}-\partial_{x_{1}^{\prime}}\right)^{n_{1}} \ldots\left(\partial_{x_{l}}-\partial_{x_{l}^{\prime}}\right)^{n_{l}} \\
& \quad \times F\left(x_{1}, \ldots, x_{l}\right) \times\left. G\left(x_{1}^{\prime}, \ldots, x_{l}^{\prime}\right)\right|_{x_{1}^{\prime}=x_{1}, \ldots, x_{l}^{\prime}=x_{l}}
\end{aligned}
$$

In the particular case, when $F=G$, formula (9) becomes

$$
\begin{aligned}
G^{-2} D_{x_{1}}^{n_{1}} \cdots D_{x_{l}}^{n_{l}} G \cdot G & =\mathscr{Y}_{n_{1} x_{1}, \ldots, n_{l} x_{l}}(0, q=2 \ln G) \\
& = \begin{cases}0, & n_{1}+\cdots+n_{l} \text { is odd }, \\
P_{n_{1} x_{1}, \ldots, n_{l} x_{l}}(q), & n_{1}+\cdots+n_{l} \text { is even, }\end{cases}
\end{aligned}
$$

in which the $P$-polynomials can be characterized by an equally recognizable even part partitional structure

$$
\begin{aligned}
& P_{2 x}(q)=q_{2 x}, \quad P_{x, t}(q)=q_{x t}, \\
& P_{4 x}(q)=q_{4 x}+3 q_{2 x}^{2}, \\
& P_{6 x}(q)=q_{6 x}+15 q_{2 x} q_{4 x}+15 q_{2 x}^{3}, \ldots
\end{aligned}
$$

This formulae will be used to obtain the bilinear Bäcklund transformations of the NLEEs. It means that once an NLEE is written in a combination form of the $\mathcal{Y}$-polynomials, then it can be easily transformed into the corresponding bilinear Bäcklund transformation form.

Theorem 2 (see [11]). The binary Bell polynomials $\mathcal{Y}_{n_{1} x_{1}, \ldots, n_{l} x_{l}}(v, w)$ can be separated into P-polynomials and $Y$-polynomials:

$$
\begin{aligned}
& (F G)^{-1} D_{x_{1}}^{n_{1}} \cdots D_{x_{l}}^{n_{l}} F \cdot G \\
& =\left.\mathscr{Y}_{n_{1} x_{1}, \ldots, n_{l} x_{l}}(v, w)\right|_{v=\ln F / G, w=\ln F G} \\
& =\left.\mathscr{Y}_{n_{1} x_{1}, \ldots, n_{l} x_{l}}(v, v+q)\right|_{v=\ln F / G, q=2 \ln G} \\
& =\sum_{n_{1}+\cdots+n_{l}=\operatorname{even}} \sum_{r_{1}=0}^{n_{1}} \cdots \sum_{r_{l}=0}^{n_{l}} \prod_{i=1}^{l}\left(\begin{array}{c}
n_{i} \\
r_{i}
\end{array}\right) P_{r_{1} x_{1}, \ldots, r_{l} x_{l}}(q) \\
& \quad \times Y_{\left(n_{1}-r_{1}\right) x_{1}, \ldots,\left(n_{l}-r_{l}\right) x_{l}}(v) .
\end{aligned}
$$

Under the Hopf-Cole transformation $v=\ln \psi$, that is, $\psi=$ $F / G$, multidimensional binary Bell polynomials $Y_{n_{1} x_{1}, \ldots, n_{l} x_{l}}(v)$ can be linearized into the following form:

$$
\left.Y_{n_{1} x_{1}, \ldots, n_{l} x_{l}}\right|_{v=\ln \psi}=\frac{\psi_{n_{1} x_{1}, \ldots, n_{l} x_{l}}}{\psi} .
$$

Formulae (13) and (14) provide the shortest way to the associated Lax system of nonlinear equations.

\section{Bilinear Representation, Bäcklund Transformation, and Conservation Laws of (1)}

In this section, we will systematically investigate bilinear representation, Bäcklund transformation, Lax pair, and infinite conservation laws of (1) based on the Bell polynomials.

3.1. Bilinear Representation. In order to detect the existence of the bilinear representation, we introduce a potential field $q$ by setting

$$
u=s q_{2 x}
$$

with $s=s(t)$ being a free function with respect to $t$, which will be chosen appropriately so that (1) is related to the $P$ polynomials. Then substituting (15) into (1) and integrating with respect to $x$ and noting condition (2) yield

$$
\begin{aligned}
E(q)= & \left(\frac{s^{\prime}(t)}{s(t)}+m(t)\right) q_{x}+q_{x t}+n(t) q_{2 x} \\
& +a(t) s(t) q_{2 x} q_{4 x}+c(t) s^{2}(t) \frac{q_{2 x}^{3}}{3}+l(t) q_{6 x}=0 .
\end{aligned}
$$

Comparing the fourth and the sixth terms of the above equation with formula (12) implies that we should require $s(t)=\rho e^{-\int m(t) d t}$. The resulting equation is then cast into a combination form of the $P$-polynomials:

$$
\begin{aligned}
E(q) & =q_{x t}+n(t) q_{2 x}+l(t)\left(q_{6 x}+15 q_{2 x} q_{4 x}+15 q_{2 x}^{3}\right) \\
& =P_{x t}(q)+n(t) P_{2 x}(q)+l(t) P_{6 x}(q)=0 .
\end{aligned}
$$

Making a change of the dependent variable

$$
q=2 \ln F \Longleftrightarrow u=s q_{2 x}=2 \rho e^{-\int m(t) d t}(\ln F)_{x x}
$$

and noting property (11), we can obtain the bilinear representation of (1) as

$$
\left(D_{x} D_{t}+l(t) D_{x}^{6}+n(t) D_{x}^{2}\right) F \cdot F=0 .
$$

Following the Hirota bilinear theory, one-soliton solution for (1) in explicit forms can be given as

$$
u_{1}=2 \rho e^{-\int m(t) d t} \frac{\partial^{2}}{\partial x^{2}}\left[\log \left(1+e^{\xi_{1}}\right)\right],
$$

and two-soliton solutions are denoted by

$$
u_{2}=2 \rho e^{-\int m(t) d t} \frac{\partial^{2}}{\partial x^{2}}\left[\log \left(1+e^{\xi_{1}}+e^{\xi_{2}}+e^{\xi_{1}+\xi_{2}+A_{12}}\right)\right]
$$

with

$$
\begin{gathered}
\xi_{j}=k_{j} x-k_{j}^{5} \int l(t) d t-k_{j} \int n(t) d t+\xi_{j}^{0}, \\
e^{A_{12}}=\frac{\left(k_{1}-k_{2}\right)^{2}\left(k_{1}^{2}-k_{1} k_{2}+k_{2}^{2}\right)}{\left(k_{1}+k_{2}\right)^{2}\left(k_{1}^{2}+k_{1} k_{2}+k_{2}^{2}\right)},
\end{gathered}
$$

where $k_{j}$ and $\xi_{j}^{0}, j=1,2$, are arbitrary real constants. 
3.2. Bäcklund Transformation and Lax Pair. In the following, we search for the bilinear Bäcklund transformation and the Lax pair of (1). Let $q^{\prime}=2 \ln F$ and $q=2 \ln G$ be two different solutions of (17), respectively; we have the two-field condition

$$
\begin{aligned}
E\left(q^{\prime}\right)-E(q)= & \left(q^{\prime}-q\right)_{x t}+n(t)\left(q^{\prime}-q\right)_{2 x}+l(t) \\
& \times\left[\left(q^{\prime}-q\right)_{6 x}+15\left(q_{2 x}^{\prime} q_{4 x}^{\prime}-q_{2 x} q_{4 x}\right)\right. \\
& \left.+15\left(q_{2 x}^{\prime 3}-q_{2 x}^{3}\right)\right]=0 .
\end{aligned}
$$

If set

$$
\begin{aligned}
& v=\frac{\left(q^{\prime}-q\right)}{2}=\ln \left(\frac{F}{G}\right), \\
& w=\frac{\left(q^{\prime}+q\right)}{2}=\ln (F G),
\end{aligned}
$$

then (24) can be rewritten as

$$
\begin{aligned}
& \frac{\left(E\left(q^{\prime}\right)-E(q)\right)}{2} \\
& =v_{x t}+n(t) v_{2 x}+l(t)\left(v_{6 x}+15 w_{4 x} v_{2 x}+15 w_{2 x} v_{4 x}\right. \\
& \left.\quad+45 v_{2 x} w_{2 x}^{2}+15 v_{2 x}^{3}\right) \\
& =\partial_{x}\left[\mathscr{Y}_{t}(v)+n(t) \mathscr{Y}_{x}(v)+l(t) \mathscr{Y}_{5 x}(v, w)\right] \\
& \quad+R(v, w)=0,
\end{aligned}
$$

with

$$
\begin{aligned}
R(v, w)=l(t) & \left(10 v_{2 x} w_{4 x}+5 v_{4 x} w_{2 x}+30 v_{2 x} w_{2 x}^{2}\right. \\
+ & 15 v_{2 x}^{3}-5 v_{x} w_{5 x}-10 v_{3 x} w_{3 x}-30 v_{x} w_{2 x} w_{3 x} \\
& -30 v_{x}^{2} v_{x x} w_{2 x}-10 v_{x}^{3} w_{3 x}-5 v_{x}^{4} v_{2 x}-20 v_{x} v_{2 x} v_{3 x} \\
& \left.-10 v_{x}^{2} v_{4 x}\right) .
\end{aligned}
$$

Taking

$$
\mathscr{Y}_{3 x}(v, w)=\lambda \text {, }
$$

where $\lambda$ is an arbitrary parameter. Then from $(28), R(v, w)$ can be rewritten in the form

$$
R(v, w)=-\frac{5}{2} l(t) \partial_{x}\left[\mathscr{Y}_{5 x}(v, w)+3 \lambda \mathscr{Y}_{3 x}(v, w)\right]
$$

Then from (26)-(29), we deduce a coupled system of $\mathcal{Y}$ polynomials:

$$
\begin{aligned}
\mathscr{Y}_{3 x}(v, w) & =\lambda, \\
\partial_{x} \mathscr{Y}_{t}(v)+\partial_{x}\left[n(t) \mathscr{Y}_{x}(v)-\frac{3}{2} l(t) \mathscr{Y}_{5 x}(v, w)\right. & \\
& \left.-\frac{15}{2} l(t) \lambda \mathscr{Y}_{2 x}(v, w)\right]=0 .
\end{aligned}
$$

By application of the identity (13), the system (30) immediately leads to the following bilinear Bäcklund transformation:

$$
\begin{aligned}
& \left(D_{x}^{3}-\lambda\right) F \cdot G=0, \\
& {\left[D_{t}+n(t) D_{x}-\frac{3}{2} l(t) D_{x}^{5}-\frac{15}{2} \lambda l(t) D_{x}^{2}+\beta\right]} \\
& \quad \times F \cdot G=0,
\end{aligned}
$$

where $\beta$ is an arbitrary parameter.

By using the Hopf-Cole transformation $v=\ln \psi$, it follows from formulas (13) and (14) that

$$
\begin{gathered}
\mathscr{Y}_{x}(v)=\frac{\psi_{x}}{\psi}, \quad \mathscr{Y}_{2 x}(v, w)=q_{2 x}+\frac{\psi_{2 x}}{\psi}, \\
\mathscr{Y}_{t}(v)=\frac{\psi_{t}}{\psi}, \quad \mathscr{Y}_{3 x}(v, w)=\frac{3 q_{2 x} \psi_{x}}{\psi}+\frac{\psi_{3 x}}{\psi}, \\
\mathscr{Y}_{5 x}(v, w)=\frac{5 q_{4 x} \psi_{x}}{\psi}+\frac{15 q_{2 x}^{2} \psi_{x}}{\psi}+\frac{10 q_{2 x} \psi_{3 x}}{\psi}+\frac{\psi_{5 x}}{\psi} ;
\end{gathered}
$$

therefore, system (30) is linearized into the corresponding Lax representation

$$
\begin{gathered}
L_{1} \psi \equiv\left(3 q_{2 x} \partial_{x}+\partial_{x}^{3}\right) \psi=\lambda \psi \\
\left(\partial_{t}+L_{2}\right) \psi \equiv\left[\partial_{t}+\left(n(t)-\frac{15}{2} l(t) q_{4 x}\right) \partial_{x}\right. \\
-\frac{15}{2} \lambda l(t) \partial_{x}^{2}-15 l(t) q_{2 x} \partial_{x}^{3}-\frac{3}{2} l(t) \partial_{x}^{5} \\
\left.+\left(-\frac{15}{2} \lambda l(t) q_{2 x}+\beta\right)\right] \psi
\end{gathered}
$$

It is easy to check that the integrability condition

$$
\left[L_{1}-\lambda, \partial_{t}+L_{2}\right] \psi=0
$$

is satisfied if $q_{2 x}=\left(e^{\int m(t) d t} / \rho\right) u$ and $u$ is a solution of the generalized variable-coefficient fifth-order Korteweg-de Vries (1).

3.3. Infinite Conservation Laws. Next, through the Bellpolynomial-type Bäcklund transformation, we will perform the procedure of deriving the infinite sequence of conservation laws of (1) in the following form:

$$
I_{n, t}+F_{n, x}=0, \quad n=1,2, \ldots
$$

Let

$$
\eta=\frac{q_{x}^{\prime}-q_{x}}{2}
$$

it follows from relation (25) that

$$
v_{x}=\eta, \quad w_{x}=q_{x}+\eta .
$$


Rewrite (30) in the conserved form

$$
\begin{gathered}
\mathscr{Y}_{3 x}(v, w)=\lambda \\
\partial_{t} \mathscr{Y}_{x}(v)+\partial_{x}\left[n(t) \mathscr{Y}_{x}(v)-\frac{3}{2} l(t) \mathscr{Y}_{5 x}(v, w)\right. \\
\left.-\frac{15}{2} \lambda l(t) \mathscr{Y}_{2 x}(v, w)\right]=0 .
\end{gathered}
$$

Substituting (37) into (38), we can obtain

$$
\begin{gathered}
\eta_{2 x}+3 \eta \eta_{x}+3 q_{2 x} \eta+\eta^{3}=\lambda=\varepsilon^{3}, \\
\eta_{t}+\partial_{x}\left[n(t) \eta-\frac{3}{2} l(t)\right. \\
\left(15 \lambda q_{2 x}+15 \lambda \eta_{x}+\eta_{4 x}+5 q_{4 x} \eta\right. \\
-15 q_{2 x}^{2} \eta+5 \eta \eta_{3 x}+5 \lambda \eta^{2}-30 q_{2 x}^{2} \eta \eta_{x} \\
\left.\left.+10 \eta^{2} \eta_{2 x}-15 \eta \eta_{x}^{2}+\eta^{5}\right)\right]=0,
\end{gathered}
$$

where we have used (39) to get (40).

To proceed, inserting the expansion

$$
\eta=\varepsilon+\sum_{n=1}^{\infty} I_{n}\left(q, q_{x}, \ldots\right) \varepsilon^{-n}
$$

into (39) and equating the coefficients for power of $\varepsilon$, we then obtain the recursion relations for the conserved densities $I_{n}$ :

$$
\begin{aligned}
& I_{1}=-q_{2 x}=-\frac{e^{\int m(t) d t}}{\rho} u, \\
& I_{2}=q_{3 x}=\frac{e^{\int m(t) d t}}{\rho} u_{x}, \\
& I_{3}=-\frac{2}{3} q_{4 x}=-\frac{2}{3} \frac{e^{\int m(t) d t}}{\rho} u_{2 x}, \\
& I_{4}=\frac{1}{3} q_{5 x}=\frac{1}{3} \frac{e^{\int m(t) d t}}{\rho} u_{3 x},
\end{aligned}
$$

and the recursion relation is given as

$$
\begin{array}{r}
I_{n}=-\frac{1}{3} I_{n-2, x x}-I_{n-1, x}-u I_{n-2}-\sum_{i=1}^{n-3}\left(I_{i} I_{n-2-i, x}\right) \\
-\sum_{i=1}^{n-2}\left(I_{i} I_{n-1-i}\right)-\frac{1}{3} \sum_{i+j+k=n-2}\left(I_{i} I_{j} I_{k}\right), \\
n=5,6,7, \ldots
\end{array}
$$

In addition, substituting (41) into (40) yields

$$
\begin{aligned}
& F_{1}=n(t) I_{1}-\frac{3}{2} l(t) \\
& \times\left[15 I_{4, x}+I_{1,4 x}+5 \frac{e^{\int m(t) d t}}{\rho} u_{2 x} I_{1}\right. \\
& -15 \frac{e^{2 \int m(t) d t}}{\rho^{2}} u^{2} I_{1}+5 I_{2,3 x} \\
& +5\left(2 I_{1} I_{3}+I_{2}^{2}+2 I_{5}\right) \\
& -30 \frac{e^{2 \int m(t) d t}}{\rho^{2}} u^{2} I_{2, x}+10\left(2 I_{1} I_{1,2 x}+I_{3,2 x}\right) \\
& \left.-15 I_{1, x}^{2}+10 I_{1}^{3}+15 I_{2}^{2}+5 I_{5}\right] \\
& F_{2}=n(t) I_{2}-\frac{3}{2} l(t) \\
& \times\left[15 I_{5, x}+I_{2,4 x}+5 \frac{e^{\int m(t) d t}}{\rho} u_{2 x} I_{2}\right. \\
& -15 \frac{e^{2 \int m(t) d t}}{\rho^{2}} u^{2} I_{2}+5\left(I_{1} I_{1,3 x}+I_{3,3 x}\right) \\
& +10\left(I_{1} I_{4}+I_{2} I_{3}+I_{6}\right)-30 \frac{e^{2 \int m(t) d t}}{\rho^{2}} \\
& \times u^{2}\left(I_{3, x}+I_{1} I_{1, x}\right)+10\left(2 I_{1} I_{2,2 x}+I_{4,2 x}\right) \\
& -30 I_{1, x} I_{2, x}+30 I_{1}^{2} I_{2}+20 I_{2} I_{3} \\
& \left.+20 I_{1} I_{4}+5 I_{6}\right] \text {, } \\
& F_{3}=n(t) I_{3}-\frac{3}{2} l(t) \\
& \times\left[15 I_{6, x}+I_{3,4 x}+5 \frac{e^{\int m(t) d t}}{\rho} u_{2 x} I_{3}\right. \\
& -15 \frac{e^{2 \int m(t) d t}}{\rho^{2}} u^{2} I_{3} \\
& +5\left(I_{1} I_{2,3 x}+I_{2} I_{1,3 x}+I_{4,3 x}\right) \\
& +5\left(2 I_{1} I_{5}+2 I_{2} I_{4}+I_{3}^{2}+2 I_{7}\right) \\
& -30 \frac{e^{2 \int m(t) d t}}{\rho^{2}} u^{2}\left(I_{4, x}+I_{1} I_{2, x}+I_{2} I_{1, x}\right) \\
& +10\left(2 I_{1} I_{3,2 x}+2 I_{3} I_{1,2 x}+I_{1}^{2} I_{1,2 x}+I_{5,2 x}\right) \\
& -15\left(I_{2, x}^{2}+I_{1} I_{2, x}^{2}\right)+5 I_{1}^{4}+30 I_{1}^{2} I_{3}
\end{aligned}
$$




$$
\begin{aligned}
& \left.+30 I_{1} I_{2}^{2}+10 I_{3}^{2}+20 I_{2} I_{4}+20 I_{1} I_{5}+5 I_{7}\right] \text {, } \\
& F_{4}=n(t) I_{4}-\frac{3}{2} l(t) \\
& \times\left[15 I_{7, x}+I_{4,4 x}+5 \frac{e^{\int m(t) d t}}{\rho} u_{2 x} I_{4}\right. \\
& -15 \frac{e^{2 \int m(t) d t}}{\rho^{2}} u^{2} I_{4} \\
& +5\left(I_{1} I_{3,3 x}+I_{2} I_{2,3 x}+I_{3} I_{1,3 x}+I_{5,3 x}\right) \\
& +10\left(2 I_{1} I_{6}+I_{2} I_{5}+I_{3} I_{4}+I_{8}\right) \\
& -30 \frac{e^{2 \int m(t) d t}}{\rho^{2}} u^{2} \\
& \times\left(I_{5, x}+I_{1} I_{3, x}+I_{3} I_{1, x}+I_{2} I_{2, x}\right) \\
& +10\left(2 I_{1} I_{4,2 x}+2 I_{1,2 x}\left(I_{1} I_{2}+I_{4}\right)\right. \\
& \left.+I_{2,2 x}\left(I_{1}^{2}+2 I_{3}\right)+I_{6,2 x}\right) \\
& -15\left(2 I_{2, x} I_{3, x}+2 I_{1, x} I_{4, x}^{2}+2 I_{1} I_{1, x} I_{2, x}\right. \\
& \left.+I_{2} I_{1, x}^{2}\right)+20 I_{1}^{3} I_{2}+60 I_{1} I_{2} I_{3} \\
& +10 I_{2}^{3}+30 I_{1}^{2} I_{4}+20 I_{2} I_{5}+20 I_{1} I_{6} \\
& \left.+20 I_{3} I_{4}+5 I_{8}\right] \\
& F_{n}=n(t) I_{n}-\frac{3}{2} l(t) \\
& \times\left[15 I_{n+3, x}+I_{n, 4 x}+5 \frac{e^{\int m(t) d t}}{\rho} u_{2 x} I_{n}\right. \\
& -15 \frac{e^{2 \int m(t) d t}}{\rho^{2}} u^{2} I_{n}+5\left(\sum_{k=1}^{n-1} I_{k} I_{n-k, 3 x}+I_{n+1,3 x}\right) \\
& +5\left(\sum_{k=1}^{n+2} I_{k} I_{n+3-k}+2 I_{n+4}\right)-30 \frac{e^{2 \int m(t) d t}}{\rho^{2}} u^{2} \\
& \times\left(\sum_{k=1}^{n-1} I_{k} I_{n-k, x}+I_{n+1, x}\right) \\
& +10 \times\left(\sum_{i+j+k=n} I_{i} I_{j} I_{k, x}+2 \sum_{k=1}^{n} I_{k} I_{n+1-k, x}+I_{n+2, x}\right) \\
& -15\left(\sum_{i+j+k=n} I_{i} I_{j, x} I_{k, x}+\sum_{k=1}^{n} I_{k, x} I_{n+1-k, x}\right) \\
& +\sum_{i+j+k+l+m=n} I_{i} I_{j} I_{k} I_{l} I_{m}+\sum_{i+j+k+l=n+1} I_{i} I_{j} I_{k} I_{l}
\end{aligned}
$$

$$
\begin{array}{r}
\left.+\sum_{i+j+k=n+2} I_{i} I_{j} I_{k}+\sum_{i+j=n+3} I_{i} I_{j}+I_{n+4}\right], \\
n=4,5,6, \ldots
\end{array}
$$

With the recursion formulae of $I_{n}$ and $F_{n}$ presented previously, the infinite conservation laws for (1) can be constructed.

\section{Quasi-Periodic Wave Solutions and Asymptotic Properties}

The quasi-periodic wave solutions of (1) are based on the following multidimensional Riemann theta function of genus $N$ :

$$
\vartheta(\xi)=\vartheta(\xi, \tau)=\sum_{n \in Z^{N}} e^{-\pi\langle\tau n, n\rangle+2 \pi i\langle\xi, n\rangle}
$$

Here the integer value vector $n=\left(n_{1}, \ldots, n_{N}\right)^{T} \in Z^{N}$, and complex phase variables $\xi=\left(\xi_{1}, \ldots, \xi_{N}\right)^{T} \in C^{N}$. Moreover, for two vectors $f=\left(f_{1}, \ldots, f_{N}\right)^{T}$ and $g=\left(g_{1}, \ldots, g_{N}\right)^{T}$, their inner product is defined by

$$
\langle f, g\rangle=f_{1} g_{1}+f_{2} g_{2}+\cdots+f_{N} g_{N}
$$

The $\tau=\left(\tau_{i j}\right)$ is a positive definite and real-valued symmetric $N \times N$ matrix, which we call the period matrix of the theta function. The entries $\tau_{i j}$ of the period matrix $\tau$ can be considered as free parameters of the theta function (45).

Now, we consider the solution for (1) in the following bilinear form:

$$
G\left(D_{x}, D_{t}\right)=\left(D_{x} D_{t}+l(t) D_{x}^{6}+n(t) D_{x}^{2}+c\right) f \cdot f=0,
$$

where $c$ is the constant of integration.

4.1. Construction of One-Periodic Waves. In this section, we consider the one-periodic wave solutions for (1). When $N=$ 1 , the theta function reduces the following Fourier series in $n$ :

$$
\vartheta(\xi, \tau)=\sum_{n=-\infty}^{\infty} e^{2 \pi i n \xi-\pi n^{2} \tau}
$$

where the phase variable $\xi=k x+\int \omega d t+\xi^{(0)}$ and the parameter $\tau>0$. 
Substituting (48) into (47), we obtain

$$
\begin{aligned}
G & \left(D_{x}, D_{t}\right) \vartheta(\xi, \tau) \cdot \vartheta(\xi, \tau) \\
& =\sum_{n=-\infty}^{\infty} \sum_{m=-\infty}^{\infty} G\left(D_{x}, D_{t}\right) e^{2 \pi i n \xi-\pi n^{2} \tau} e^{2 \pi i m \xi-\pi m^{2} \tau} \\
& =\sum_{n=-\infty}^{\infty} \sum_{m=-\infty}^{\infty} G[2 \pi i(n-m) k, 2 \pi i(n-m) \omega] \\
& \times e^{2 \pi(n+m) \xi-\pi\left(n^{2}+m^{2}\right) \tau} \\
= & \sum_{m^{\prime}=-\infty}^{\infty}\left\{\sum _ { n = - \infty } ^ { \infty } G \left[2 \pi i\left(2 n-m^{\prime}\right) k,\right.\right. \\
= & \sum_{m^{\prime}=-\infty}^{\infty} \bar{G}\left(m^{\prime}\right) e^{2 \pi i m^{\prime} \xi},
\end{aligned}
$$

where

$$
\begin{aligned}
\bar{G}\left(m^{\prime}\right)=\sum_{n=-\infty}^{\infty} & G\left[2 \pi i\left(2 n-m^{\prime}\right) k, 2 \pi i\left(2 n-m^{\prime}\right) \omega\right] \\
& \times e^{-\pi\left[n^{2}+\left(n-m^{\prime}\right)^{2}\right] \tau} .
\end{aligned}
$$

By shifting sum index as $n=n^{\prime}+1$, we conclude that

$$
\begin{aligned}
\bar{G}\left(m^{\prime}\right)= & \left(\sum _ { n ^ { \prime } = - \infty } ^ { \infty } G \left\{2 \pi i\left[2 n^{\prime}-\left(m^{\prime}-2\right)\right] k,\right.\right. \\
\left.2 \pi i\left[2 n^{\prime}-\left(m^{\prime}-2\right)\right] \omega\right\} & \\
& \left.\times e^{-\pi\left\{n^{\prime 2}+\left[n^{\prime}-\left(m^{\prime}-2\right)\right]^{2}\right\} \tau}\right) e^{\left[-2 \pi\left(m^{\prime}-1\right) \tau\right]} \\
= & \bar{G}\left(m^{\prime}-2\right) e^{-2 \pi\left(m^{\prime}-1\right) \tau}=\cdots \\
= & \begin{cases}\bar{G}(0) e^{-\pi m^{\prime 2}(\tau / 2)}, & m^{\prime} \text { is even, } \\
\bar{G}(1) e^{-\pi\left(m^{\prime 2}-1\right)(\tau / 2)}, & m^{\prime} \text { is odd, }\end{cases}
\end{aligned}
$$

which imply that if $\bar{G}(0)=\bar{G}(1)=0$, then it follows that

$$
\bar{G}\left(m^{\prime}\right)=0, \quad m^{\prime} \in Z,
$$

and thus the theta function (48) is the exact solution of (47). In this way, we may let

$$
\begin{aligned}
& \bar{G}(0)=\sum_{n=-\infty}^{\infty}[-16 \pi^{2} n^{2} k \omega-4096 \pi^{6} n^{6} k^{6} l(t) \\
&\left.-16 \pi^{2} n^{2} k^{2} n(t)+c\right] e^{-2 \pi n^{2} \tau}=0, \\
& \bar{G}(1)=\sum_{n=-\infty}^{\infty}\left[-4 \pi^{2}(2 n-1)^{2} k \omega-64 \pi^{6}(2 n-1)^{6} k^{6} l(t)\right. \\
&\left.-4 \pi^{2}(2 n-1)^{2} k^{2} n(t)+c\right] e^{-\pi\left(2 n^{2}-2 n+1\right) \tau}=0 .
\end{aligned}
$$

Denote

$$
\begin{aligned}
& \lambda=e^{-\pi \tau}, \quad a_{11}=-\sum_{n=-\infty}^{\infty} 16 \pi^{2} n^{2} k \lambda^{2 n^{2}}, \\
& a_{12}= \sum_{n=-\infty}^{\infty} \lambda^{2 n^{2}}, \quad a_{22}=\sum_{n=-\infty}^{\infty} \lambda^{2 n^{2}-2 n+1}, \\
& a_{21}=-\sum_{n=-\infty}^{\infty} 4 \pi^{2}(2 n-1)^{2} k \lambda^{2 n^{2}-2 n+1}, \\
& b_{1}=\sum_{n=-\infty}^{\infty}\left(4096 \pi^{6} n^{6} k^{6} l(t)+16 \pi^{2} n^{2} k^{2} n(t)\right) \lambda^{2 n^{2}}, \\
& b_{2}=\sum_{n=-\infty}^{\infty}\left(64 \pi^{6}(2 n-1)^{6} k^{6} l(t)+4 \pi^{2}(2 n-1)^{2}\right. \\
&\left.\quad \times k^{2} n(t)\right) \lambda^{2 n^{2}-2 n+1} .
\end{aligned}
$$

Then (53) can be written as

$$
\left(\begin{array}{ll}
a_{11} & a_{12} \\
a_{21} & a_{22}
\end{array}\right)\left(\begin{array}{l}
\omega \\
c
\end{array}\right)=\left(\begin{array}{l}
b_{1} \\
b_{2}
\end{array}\right) .
$$

Notice that there are a lot of choices for the angular wave number $k$. The determinant of the coefficient matrix $A(k)=$ $\left(a_{i j}(k)\right)_{2 \times 2}$ is a polynomial in $k$; if $\operatorname{det}(A(k)) \not \equiv 0$, then

$$
A_{0}:=\{k \in R \mid \operatorname{det}(A(k))=0\}
$$

is either an empty set or a finite set, and so, there are real solutions $(\omega, c)$ to the system (55) for $k \notin A_{0}$. Solving this system, we have

$$
\omega=\frac{b_{1} a_{22}-b_{2} a_{12}}{a_{11} a_{22}-a_{12} a_{21}} .
$$

Therefore we get a one-periodic wave solution of (1):

$$
u=2 \rho e^{-\int m(t) d t}(\log \vartheta(\xi, \tau))_{x x}
$$

where the parameter $\omega$ is given by (57). 
4.2. Asymptotic Property of One-Periodic Waves. In the following, we further consider asymptotic properties of the oneperiodic wave solution. It is shown that the soliton solution of (1) can be obtained as a limit of the one-periodic wave solution. The relation between these two solutions can be established as Theorem 3 .

Theorem 3. Suppose that the vector $(\omega, c)^{T}$ is a solution of the system (55), and for the quasi-periodic wave solution (58), we let

$$
\xi=\frac{\xi^{\prime}}{2 \pi i}+\frac{\tau}{2 i}, \quad \xi^{\prime}=k^{\prime} x+\int \omega^{\prime} d t+\xi^{(0)^{\prime}},
$$

where $k^{\prime}=2 \pi i k, \omega^{\prime}=2 \pi i \omega, \xi^{(0)^{\prime}}$ and $=2 \pi i \xi^{(0)}-\pi \tau$. Then the one-periodic solution (58) tends to the one-soliton solution (20) under a small amplitude limit; that is,

$$
u \longrightarrow u_{1}, \quad \text { as } \lambda \longrightarrow 0
$$

Proof. By using (54), we write functions $a_{i j}, b_{j}, i, j=1,2$, as the series about $\lambda$ :

$$
\begin{aligned}
& a_{11}=-32 \pi^{2} k\left(\lambda^{2}+4 \lambda^{8}+9 \lambda^{18}+\cdots\right), \\
& a_{12}=1+2 \lambda^{2}+2 \lambda^{8}+2 \lambda^{18}+2 \lambda^{32}+\cdots, \\
& a_{21}=-8 \pi^{2} k\left(\lambda+9 \lambda^{5}+25 \lambda^{13}+\cdots\right), \\
& a_{22}=2 \lambda+2 \lambda^{5}+2 \lambda^{13}+2 \lambda^{25}+\cdots, \\
& b_{1}=\left(8192 \pi^{6} k^{6} l(t)+32 \pi^{2} k^{2} n(t)\right) \lambda^{2}+\cdots, \\
& b_{2}=\left(128 \pi^{6} k^{6} l(t)+8 \pi^{2} k^{2} n(t)\right) \lambda+\cdots .
\end{aligned}
$$

Suppose that the solution of system (55) has the following form:

$$
\omega=\omega_{0}+\omega_{1} \lambda^{1}+\omega_{2} \lambda^{2}+\cdots=\omega_{0}+o(\lambda),
$$

substituting expansions (61) and (62) into system (55), and let $\lambda \rightarrow 0$; we can obtain the following relation immediately:

$$
\omega_{0}=-16 \pi^{4} k^{5} l(t)-k n(t)
$$

combining (59) and (63), we then obtain

$$
\begin{aligned}
\omega^{\prime} & =2 \pi i \omega \longrightarrow-32 \pi^{5} l(t) i k^{5}-2 \pi n(t) i k, \\
& =-l(t) k^{\prime 5}-n(t) k^{\prime} .
\end{aligned}
$$

It remains to show that the one-periodic wave (58) degenerates to the one-soliton solution (20) under the limit $\lambda \rightarrow 0$.
We first expand the periodic function $\vartheta(\xi)$ in the form

$$
\vartheta(\xi, \tau)=1+\lambda\left(e^{2 \pi i \xi}+e^{-2 \pi i \xi}\right)+\lambda^{4}\left(e^{4 \pi i \xi}+e^{-4 \pi i \xi}\right)+\cdots
$$

By using transformation (59), it follows that

$$
\begin{aligned}
\vartheta(\xi, \tau) & =1+e^{\xi^{\prime}}+\lambda^{2}\left(e^{-\xi^{\prime}}+e^{2 \xi^{\prime}}\right)+\lambda^{6}\left(e^{-2 \xi^{\prime}}+e^{3 \xi^{\prime}}\right)+\cdots, \\
& \longrightarrow 1+e^{\xi^{\prime}}, \quad \text { as } \lambda \longrightarrow 0 .
\end{aligned}
$$

Thus, we conclude that the one-periodic solution (58) may go to one-soliton solution (20) as the amplitude $\lambda \rightarrow 0$.

4.3. Construction of Two-Periodic Waves. In the case when $N=2$, the Riemann theta function (45) takes the form

$$
\vartheta(\xi, \tau)=\vartheta\left(\xi_{1}, \xi_{2}, \tau\right)=\sum_{n \in Z^{2}} e^{-\pi\langle\tau n, n\rangle+2 \pi i\langle\xi, n\rangle},
$$

where $n=\left(n_{1}, n_{2}\right)^{T} \in Z^{2}, \xi=\left(\xi_{1}, \xi_{2}\right)^{T} \in C^{2}$, and $\xi_{j}=k_{j} x+$ $\int \omega_{j} d t+\xi_{j}^{(0)}, j=1,2 . \tau$ is a positive definite and real-valued symmetric $2 \times 2$ matrix which can take the form

$$
\tau=\left(\begin{array}{ll}
\tau_{11} & \tau_{12} \\
\tau_{21} & \tau_{22}
\end{array}\right), \quad \tau_{11}>0, \tau_{22}>0, \tau_{11} \tau_{22}-\tau_{12}^{2}>0
$$

Substituting (67) into (47), we have

$$
\begin{aligned}
G\left(D_{x}, D_{t}\right) \vartheta\left(\xi_{1}, \xi_{2}, \tau\right) \cdot \vartheta\left(\xi_{1}, \xi_{2}, \tau\right) \\
\quad=\sum_{m, n \in Z^{2}} G(2 \pi i\langle n-m, k\rangle, 2 \pi i\langle n-m, \omega\rangle)
\end{aligned}
$$

$$
\times e^{2 \pi i\langle\xi, n+m\rangle-\pi(\langle\tau m, m\rangle+\langle\tau n, n\rangle)}
$$

$$
\begin{gathered}
\stackrel{m=m^{\prime}-n}{=} \sum_{m^{\prime} \in Z^{2}} \sum_{n \in Z^{2}} G\left(2 \pi i\left\langle 2 n-m^{\prime}, k\right\rangle, 2 \pi i\left\langle 2 n-m^{\prime}, \omega\right\rangle\right) \\
\times e^{-\pi\left[\left\langle\tau\left(n-m^{\prime}, n-m^{\prime}\right\rangle\right]+\langle\tau n, n\rangle\right]} \cdot e^{2 \pi i\left\langle\xi, m^{\prime}\right\rangle} \\
=\sum_{m^{\prime} \in Z^{2}} \bar{G}\left(m_{1}^{\prime}, m_{2}^{\prime}\right) e^{2 \pi i\left\langle m^{\prime}, \xi\right\rangle},
\end{gathered}
$$


where $\bar{G}\left(m_{1}^{\prime}, m_{2}^{\prime}\right)$ is the coefficient of $e^{2 \pi i\left\langle m^{\prime}, \xi\right\rangle}$. For each fixed $l=1,2$, by shifting $j$ th summation index as $n_{j}=n_{j}^{\prime}+\delta_{j, l}$ with $\delta_{j, l}$ representing Kronecker's delta we obtain that

$$
\begin{gathered}
\bar{G}\left(m_{1}^{\prime}, m_{2}^{\prime}\right) \\
=\sum_{n \in Z^{2}} G\left(2 \pi i\left\langle 2 n-m^{\prime}, k\right\rangle, 2 \pi i\left\langle 2 n-m^{\prime}, \omega\right\rangle\right) \\
\times e^{-\pi\left[\left\langle\tau\left(n-m^{\prime}, n-m^{\prime}\right\rangle\right]+\langle\tau n, n\rangle\right]} \\
=\sum_{n^{\prime} \in Z^{2}} G\left(2 \pi i \sum_{j=1}^{2}\left[2 n_{j}^{\prime}-\left(m_{j}^{\prime}-2 \delta_{j l}\right)\right] k_{j},\right. \\
\times \exp \left\{\begin{array}{c}
\left.2 \pi i \sum_{j=1}^{2}\left[2 n_{j}^{\prime}-\left(m_{j}^{\prime}-2 \delta_{i j}\right)\right] \omega_{j}\right) \\
-\pi \sum_{j, h=1}^{2}\left(n_{j}^{\prime}+\delta_{j l}\right) \tau_{j h}\left(n_{h}^{\prime}+\delta_{h l}\right) \\
-\pi \sum_{j, h=1}^{2}\left[\left(m_{j}^{\prime}+2 \delta_{j l}-n_{j}^{\prime}\right)+\delta_{j l}\right] \\
\times \tau_{j h}\left[\left(m_{h}^{\prime}-2 \delta_{h l}-n_{h}^{\prime}\right)+\delta_{h l}\right]
\end{array}\right\} \\
=\left\{\begin{array}{c}
\bar{G}\left(m_{1}^{\prime}-2, m_{2}^{\prime}\right) e^{-2 \pi\left(\tau_{11} m_{1}^{\prime}+\tau_{12} m_{2}^{\prime}\right)+2 \pi \tau_{11}}, \quad l=1, \\
\bar{G}\left(m_{1}^{\prime}, m_{2}^{\prime}-2\right) e^{-2 \pi\left(\tau_{12} m_{1}^{\prime}+\tau_{22} m_{2}^{\prime}\right)+2 \pi \tau_{22}}, \quad l=2,
\end{array}\right.
\end{gathered}
$$

which implies that if the following equations are satisfied

$$
\bar{G}(0,0)=\bar{G}(0,1)=\bar{G}(1,0)=\bar{G}(1,1)=0
$$

then we have $\bar{G}\left(m_{1}^{\prime}, m_{2}^{\prime}\right)=0$, for all $m_{1}^{\prime}, m_{2}^{\prime} \in Z$, and thus the function (67) is an exact solution of (47). By introducing the notations as

$$
\begin{aligned}
A= & \left(a_{j l}\right)_{4 \times 3^{\prime}}, \quad b=\left(b_{1}, b_{2}, b_{3}, b_{4}\right)^{T}, \\
a_{j 1}= & -4 \pi^{2} \sum_{n_{1}, n_{2} \in Z^{2}}\left\langle 2 n-s^{j}, k\right\rangle\left(2 n_{1}-s_{1}^{j}\right) \varepsilon_{j}(n), \\
a_{j 2}= & -4 \pi^{2} \sum_{n_{1}, n_{2} \in Z^{2}}\left\langle 2 n-s^{j}, k\right\rangle\left(2 n_{2}-s_{2}^{j}\right) \varepsilon_{j}(n), \\
a_{j 3}= & \sum_{n_{1}, n_{2} \in Z^{2}} \varepsilon_{j}(n), \\
b_{j}= & -l(t)(2 \pi i)^{6}\left\langle 2 n-s^{j}, k\right\rangle^{6} \\
& -n(t)(2 \pi i)^{2} \times\left\langle 2 n-s^{j}, k\right\rangle^{2},
\end{aligned}
$$

$$
\begin{array}{rlrl}
\varepsilon_{j}(n) & =\lambda_{1}^{n_{1}^{2}+\left(n_{1}-s_{1}^{j}\right)^{2}} \lambda_{2}^{n_{2}^{2}+\left(n_{2}-s_{2}^{j}\right)^{2}} \lambda_{1}^{n_{1} n_{2}+\left(n_{1}-s_{1}^{j}\right)\left(n_{2}-s_{2}^{j}\right),} \\
\lambda_{1} & =e^{-\pi \tau_{11}}, & & \lambda_{2}=e^{-\pi \tau_{22}}, \quad \lambda_{3}=e^{-2 \pi \tau_{12},} \\
s^{j} & =\left(s_{1}^{j}, s_{2}^{j}\right), & & j=1,2,3,4, \\
s^{1} & =(0,0), & & s^{2}=(1,0), \\
s^{3} & =(0,1), & & s^{4}=(1,1),
\end{array}
$$

(71) can be written as a linear system:

$$
A\left(\omega_{1}, \omega_{2}, c\right)^{T}=b .
$$

Notice that both $A$ and $b$ depend on $k$, so the solution of (73) also depends on $k$. And if

$$
\operatorname{rank}(A(k))=\operatorname{rank}(A(k), b(k))=3,
$$

then there is a unique nonzero solution of $\left(\omega_{1}, \omega_{2}, c\right)$ to the system (73). Solving this system, we can get a two-periodic wave solution of (1):

$$
u=2 \rho e^{-\int m(t) d t}\left(\log \vartheta\left(\xi_{1}, \xi_{2}, \tau\right)\right)_{x x},
$$

with $\vartheta\left(\xi_{1}, \xi_{2}\right)$ and $\omega_{1}, \omega_{2}, c$ given by (67) and (73), respectively, while $k=\left(k_{1}, k_{2}\right)$ is determined by $(74)$.

4.4. Asymptotic Property of Two-Periodic Waves. In this subsection, we consider the asymptotic properties of the twoperiodic solution (75). In a similar way to Theorem 3, we can establish the relation between the two-periodic solution (75) and the two-soliton solution (21) as follows.

Theorem 4. Suppose that the vector $\left(\omega_{1}, \omega_{2}, c\right)^{T}$ is a solution of the system (73), and for the quasi-periodic wave solution (75), one lets

$$
\xi_{j}=\frac{\xi_{j}^{\prime}}{2 \pi i}+\frac{\tau_{j j}}{2 i}, \quad \xi_{j}^{\prime}=k_{j}^{\prime} x+\int \omega_{j}^{\prime} d t+\xi_{j}^{(0)^{\prime}},
$$

where $k_{j}^{\prime}=2 \pi i k_{j}, \omega_{j}^{\prime}=2 \pi i \omega_{j}, \xi_{j}^{(0)^{\prime}}=2 \pi i \xi_{j}^{(0)}-\pi \tau_{j j}$, and $\tau_{12}=A_{12} / 2 \pi, j=1,2$, and $A_{12}$ is given in (21). Then the two-periodic solution (75) tends to the two-soliton solution (21) under a small amplitude limit; that is,

$$
u \longrightarrow u_{2}, \quad \text { as } \lambda_{1}, \lambda_{2} \longrightarrow 0 .
$$

Proof. According to formula (67), we expand the function $\vartheta\left(\xi_{1}, \xi_{2}\right)$ in the following form:

$$
\begin{aligned}
\vartheta\left(\xi_{1}, \xi_{2}, \tau\right)= & +\left(e^{2 \pi i \xi_{1}}+e^{-2 \pi i \xi_{1}}\right) e^{-\pi \tau_{11}} \\
& +\left(e^{2 \pi i \xi_{2}}+e^{-2 \pi i \xi_{2}}\right) e^{-\pi \tau_{22}} \\
& +\left(e^{2 \pi i\left(\xi_{1}+\xi_{2}\right)}+e^{-2 \pi i\left(\xi_{1}+\xi_{2}\right)}\right) \\
& \times e^{-\pi\left(\tau_{11}+2 \tau_{12}+\tau_{22}\right)}+\cdots .
\end{aligned}
$$


By using (76), we get

$$
\begin{aligned}
& \vartheta\left(\xi_{1}, \xi_{2}, \tau\right)= 1+e^{\xi_{1}^{\prime}}+e^{\xi_{2}^{\prime}}+e^{\xi_{1}^{\prime}+\xi_{2}^{\prime}-2 \pi \tau_{12}} \\
&+\lambda_{1}^{2} e^{-\xi_{1}^{\prime}}+\lambda_{2}^{2} e^{-\xi_{2}^{\prime}} \\
&+\lambda_{1}^{2} \lambda_{2}^{2} e^{-\xi_{1}^{\prime}-\xi_{2}^{\prime}-2 \pi \tau_{12}}+\cdots \\
& \longrightarrow 1+e^{\xi_{1}^{\prime}}+e^{\xi_{2}^{\prime}}+e^{\xi_{1}^{\prime}+\xi_{2}^{\prime}+A_{12}}, \\
& \text { as } \lambda_{1}, \lambda_{2} \longrightarrow 0
\end{aligned}
$$

where $\xi_{j}^{\prime}=k_{j}^{\prime} x+\int \omega_{j}^{\prime} d t+\xi_{j}^{(0)^{\prime}}, j=1,2$. Thus the two-periodic wave solutions can be reduced to two-soliton solutions (21) under the limit $\lambda_{1}, \lambda_{2} \rightarrow 0$; we only need to prove that

$$
\omega_{j}^{\prime} \longrightarrow-l(t) k_{j}^{\prime 5}-n(t) k_{j}^{\prime}, \quad j=1,2 .
$$

And the proof of (80) is similar to the proof of formula (64).

\section{Conclusions}

In this paper, the generalized variable-coefficient fifth-order Korteweg-de Vries equation is investigated. By virtue of the Bell-polynomial approach, bilinear form of (1) has been derived under condition (2) and bilinear Bäcklund transformation, Lax pairs, and infinite conservation laws of the equation are constructed. Furthermore, the Riemann theta functions have been used to generate one-periodic and two-periodic wave solutions of the equation, and the relations between the periodic wave solutions and soliton solutions are also established. The quasi-periodic solutions play an important role in understanding the diversity and integrability of nonlinear differential equations. We think that there are still many deep relations between the quasi-periodic solutions and other kinds of solutions which still remain open and worth studying.

\section{Conflict of Interests}

The authors declare that there is no conflict of interests regarding the publication of this paper.

\section{Acknowledgments}

This work was supported by the Fundamental Research Funds for the Central Universities (no. 2013XK03) and the National Natural Science Foundation of China (Grant no. 11371361).

\section{References}

[1] M. J. Ablowitz and P. A. Clarkson, Solitons, Nonlinear Evolution Equations and Inverse Scattering, Cambridge University Press, New York, NY, USA, 1991.

[2] V. B. Matveev and M. A. Salle, Darboux Transformations and Solitons, Springer, Berlin, Germany, 1991.
[3] C. H. Gu, H. S. Hu, and Z. X. Zhou, Darboux Transformation in Solitons Theory and Geometry Applications, Shanghai Science and Technology Press, Shanghai, China, 1999.

[4] T. C. Xia, X. H. Chen, and D. Y. Chen, "Darboux transformation and soliton-like solutions of nonlinear Schrödinger equations," Chaos, Solitons \& Fractals, vol. 26, no. 3, pp. 889-896, 2005.

[5] M. R. Miura, Bäcklund Transformation, Springer, Berlin, Germany, 1978.

[6] Y. Chen, Z. Y. Yan, and H. Q. Zhang, "Exact solutions for a family of variable-coefficient "reaction-duffing" equations via the Bäcklund transformation," Theoretical and Mathematical Physics, vol. 132, no. 1, pp. 970-975, 2002.

[7] R. Hirota, The Direct Method in Soliton Theory, Cambridge University Press, New York, NY, USA, 2004.

[8] A.-M. Wazwaz, "The Hirota's direct method for multiple-soliton solutions for three model equations of shallow water waves," Applied Mathematics and Computation, vol. 201, no. 1-2, pp. 489-503, 2008.

[9] E. D. Belokolos, A. I. Bobenko, V. Z. Enolskij, A. R. Its, and V. B. Matveev, Algebro-Geometric Approach to Nonlinear Integrable Equations, Springer, Berlin, Germany, 1994.

[10] F. Gesztesy and H. Holden, Soliton Equations and Their AlgebroGeometric Solutions-I: (1+1)-Dimensional Continuous Models, Cambridge University Press, New York, NY, USA, 2003.

[11] F. Gesztesy, H. Holden, J. Michor, and G. Teschl, Soliton Equations and Their Algebro-Geometric Solutions-II: $(1+1)$ Dimensional Discrete Models, Cambridge University Press, Cambridge, New York, NY, USA, 2008.

[12] C. Gilson, F. Lambert, J. Nimmo, and R. Willox, "On the combinatorics of the Hirota D-operators," Proceedings of the Royal Society A: Mathematical, Physical \& Engineering Sciences, vol. 452, no. 1945, pp. 223-234, 1996.

[13] F. Lambert, I. Loris, and J. Springael, "Classical Darboux transformations and the KP hierarchy," Inverse Problems, vol. 17, no. 4, pp. 1067-1074, 2001.

[14] F. Lambert and J. Springael, "Soliton equations and simple combinatorics," Acta Applicandae Mathematicae, vol. 102, no. 23, pp. 147-178, 2008.

[15] E. G. Fan, "The integrability of nonisospectral and variablecoefficient KdV equation with binary Bell polynomials," Physics Letters A, vol. 375, no. 3, pp. 493-497, 2011.

[16] Y. C. Hon and E. G. Fan, "Binary Bell polynomial approach to the non-isospectral and variable-coefficient KP equations," IMA Journal of Applied Mathematics, vol. 77, no. 2, pp. 236-251, 2012.

[17] E. G. Fan and K. W. Chow, "Darboux covariant Lax pairs and infinite conservation laws of the $(2+1)$-dimensional breaking soliton equation," Journal of Mathematical Physics, vol. 52, no. 2, Article ID 023504, 2011.

[18] E. G. Fan and Y. C. Hon, "Super extension of Bell polynomials with applications to supersymmetric equations," Journal of Mathematical Physics, vol. 53, no. 1, Article ID 013503, 2012.

[19] E. G. Fan, "New bilinear Bäcklund transformation and Lax pair for the supersymmetric two-Boson equation," Studies in Applied Mathematics, vol. 127, no. 3, pp. 284-301, 2011.

[20] W. X. Ma, "Bilinear equations, Bell polynomials and linear superposition principle," Journal of Physics: Conference Series, vol. 411, no. 1, Article ID 012021, 2013.

[21] A. Nakamura, "A direct method of calculating periodic wave solutions to nonlinear evolution equations-I. Exact twoperiodic wave solution," Journal of the Physical Society of Japan, vol. 47, no. 5, pp. 1701-1705, 1979. 
[22] A. Nakamura, "A direct method of calculating periodic wave solutions to nonlinear evolution equations-II. Exact one- and two-periodic wave solution of the coupled bilinear equations," Journal of the Physical Society of Japan, vol. 48, no. 4, pp. 13651370, 1980.

[23] Y. C. Hon, E. G. Fan, and Z. Qin, "A kind of explicit quasiperiodic solution and its limit for the TODA lattice equation," Modern Physics Letters B, vol. 22, no. 8, pp. 547-553, 2008.

[24] E. G. Fan and Y. C. Hon, "Quasi-periodic waves and asymptotic behavior for Bogoyavlenskiis breaking soliton equation in $(2+1)$ dimensions," Physical Review E, vol. 78, no. 3, Article ID 036607, 13 pages, 2008.

[25] E. G. Fan, "Quasi-periodic waves and an asymptotic property for the asymmetrical Nizhnik-Novikov-Veselov equation," Journal of Physics A : Mathematical and Theoretical, vol. 42, no. 9, Article ID 095206, 2009.

[26] W.-X. Ma, R. G. Zhou, and L. Gao, "Exact one-periodic and twoperiodic wave solutions to Hirota bilinear equations in $(2+1)$ dimensions," Modern Physics Letters A, vol. 24, no. 21, pp. 16771688, 2009.

[27] Y. Zhang, Z. L. Cheng, and X. H. Hong, "Riemann theta function periodic wave solutions for the variable-coefficient mKdV equation," Chinese Physics B, vol. 21, no. 12, Article ID 120203, 2012.

[28] M. Zamir, The Physics of Pulsatile Flow, Springer, New York, NY, USA, 2000.

[29] F. Dalfovo, S. Giorgini, L. P. Pitaevskii, and S. Stringari, "Theory of Bose-Einstein condensation in trapped gases," Reviews of Modern Physics, vol. 71, no. 3, pp. 463-512, 1999.

[30] B. Tian, G. M. Wei, C. Y. Zhang, W. R. Shan, and Y. T. Gao, "Transformations for a generalized variable-coefficient Korteweg-de Vries model from blood vessels, Bose-Einstein condensates, rods and positons with symbolic computation," Physics Letters A, vol. 356, no. 1, pp. 8-16, 2006.

[31] V. N. Serkin and A. Hasegawa, "Novel soliton solutions of the nonlinear Schrödinger equation model," Physical Review Letters, vol. 85, no. 21, pp. 4502-4505, 2000.

[32] V. I. Kruglov, A. C. Peacock, and J. D. Harvey, "Exact solutions of the generalized nonlinear Schrödinger equation with distributed coefficients," Physical Review E, vol. 71, no. 5, Article ID 056619, 2005.

[33] Z. Y. Yan, "Nonautonomous "rogons" in the inhomogeneous nonlinear Schrödinger equation with variable coefficients," Physics Letters A, vol. 374, no. 4, pp. 672-679, 2010.

[34] F. C. You, T. C. Xia, and J. Zhang, "Frobenius integrable decompositions for two classes of nonlinear evolution equations with variable coefficients," Modern Physics Letters B, vol. 23, no. 12, pp. 1519-1524, 2009.

[35] X.-Y. Tang, Y. Gao, F. Huang, and S.-Y. Lou, "Variable coefficient nonlinear systems derived from an atmospheric dynamical system," Chinese Physics B, vol. 18, no. 11, pp. 4622-4635, 2009.

[36] X. Y. Yu, Y.-T. Gao, Z.-Y. Sun, and Y. Liu, "N-soliton solutions, Bäcklund transformation and Lax pair for a generalized variable-coefficient fifth-order Korteweg-de Vries equation," Physica Scripta, vol. 81, no. 4, Article ID 045402, 2010.

[37] J. Weiss, M. Tabor, and G. Carnevale, "The Painlevé property for partial differential equations," Journal of Mathematical Physics, vol. 24, no. 3, pp. 522-526, 1983.

[38] B. Chen and Y. C. Xie, "An auto-Bäcklund transformation and exact solutions of stochastic Wick-type Sawada-Kotera equations," Chaos, Solitons \& Fractals, vol. 23, no. 1, pp. 243248, 2005. 


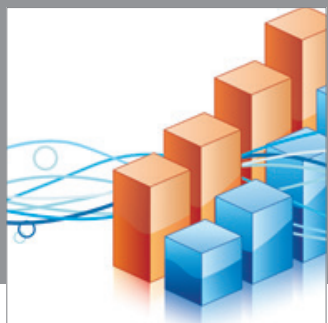

Advances in

Operations Research

mansans

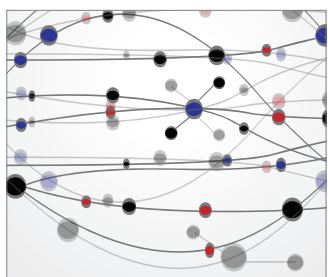

The Scientific World Journal
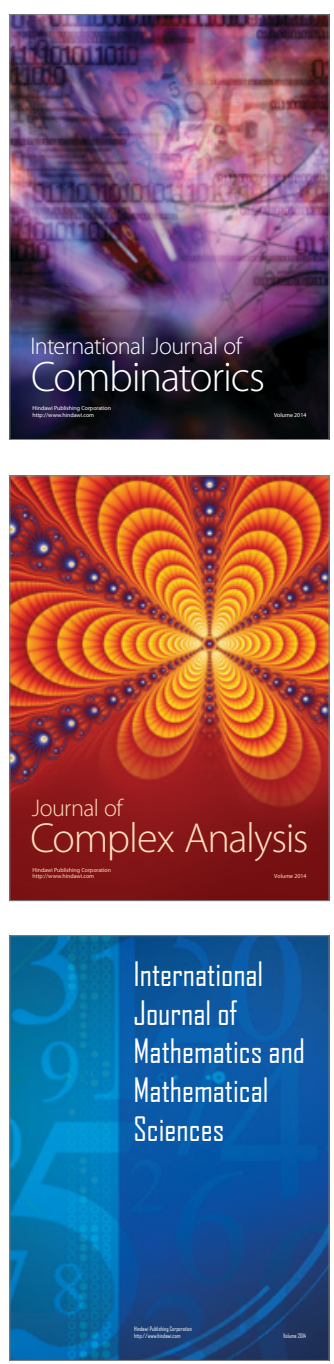
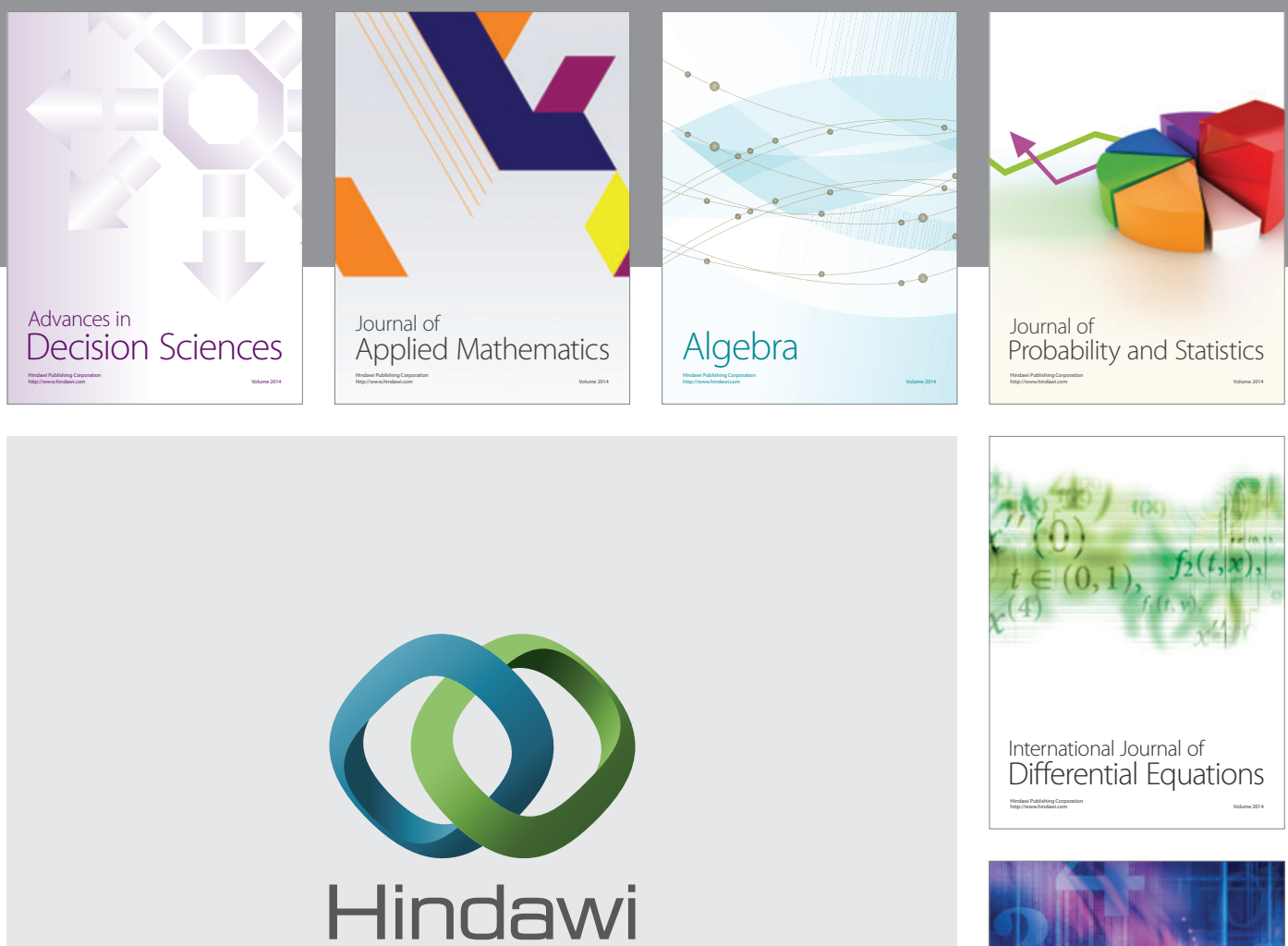

Submit your manuscripts at http://www.hindawi.com
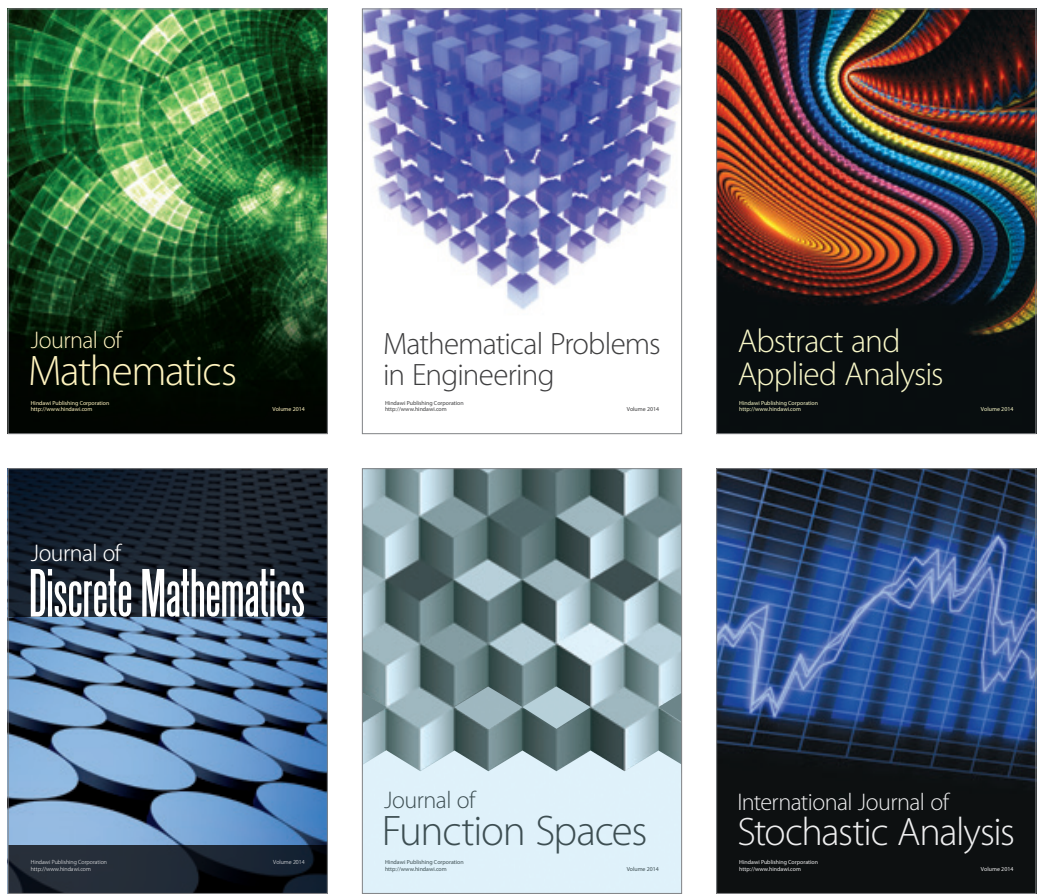

Journal of

Function Spaces

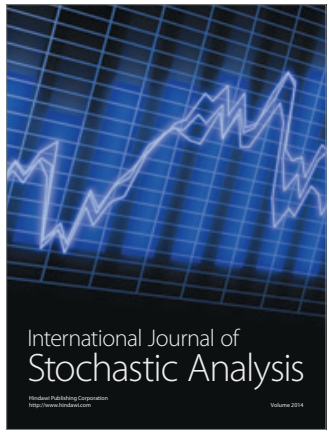

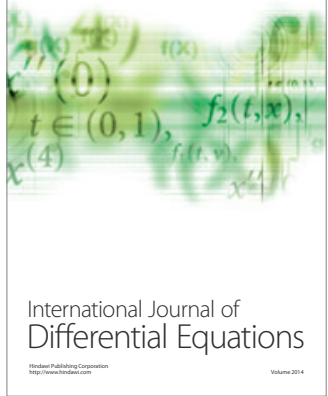
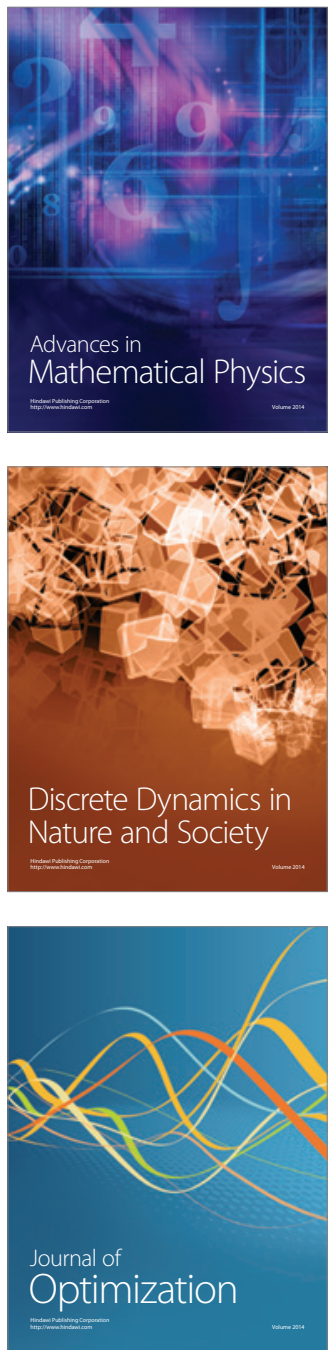